\title{
A unified materials approach to mitigating optical nonlinearities in fiber lasers
}

John Ballato, Maxime Cavillon, Peter Dragic, Courtney Kucera, Thomas Hawkins

John Ballato, Maxime Cavillon, Peter Dragic, Courtney Kucera, Thomas Hawkins, "A unified materials approach to mitigating optical nonlinearities in fiber lasers," Proc. SPIE 10683, Fiber Lasers and Glass Photonics: Materials through Applications, 1068302 (17 May 2018); doi: 10.1117/12.2307163 


\title{
A Unified Materials Approach to Mitigating Optical Nonlinearities in Fiber Lasers
}

John Ballato ${ }^{\mathrm{a}, *}$, Maxime Cavillon ${ }^{\mathrm{a}}$, Peter Dragic ${ }^{\mathrm{b}}$, Courtney Kucera ${ }^{\mathrm{a}}$, and Thomas Hawkins ${ }^{\mathrm{a}}$, ${ }^{\mathrm{a}}$ The Center for Optical Materials Science and Engineering Technologies (COMSET) and the Department of Materials Science and Engineering, Clemson University, Clemson, SC 29634 USA;

${ }^{\mathrm{b}}$ Department of Electrical and Computer Engineering, University of Illinois at Urbana Champaign, Urbana, IL Urbana, IL 61801 USA

* Corresponding author: jballat@clemson.edu

\begin{abstract}
This paper provides a road-map for the development of simple core/clad optical fibers whose enhanced performance - in particular, marked reductions in optical nonlinearities - is achieved materially and not through the more conventional present routes of geometrically complex fiber design. More specifically, the material properties that give rise to Brillouin, Raman, and Rayleigh scattering, transverse mode instabilities (TMI), and $\mathrm{n}_{2}$-mediated nonlinear effects are compiled and results on a wide range of optical fibers are discussed with a focus on trends in performance with glass composition. Further, optical power scaling estimations as well as binary and ternary property diagrams associated with Rayleigh scattering, the Brillouin gain coefficient (BGC) and the thermo-optic coefficient $(\mathrm{dn} / \mathrm{dT})$ are developed and employed to graphically represent general trends with composition along with compositional targets for a single intrinsically low nonlinearity, silica-based optical fiber that can achieve the powerscaling goals of future high energy fiber laser applications.
\end{abstract}

Keywords: Fiber lasers, optical fiber, optical nonlinearities, stimulated Brillouin scattering, Raman scattering, transverse mode instability

\section{INTRODUCTION}

Optical nonlinearities are the present and future limitations plaguing scaling to higher output powers in modern high energy laser (HEL) systems [1]. Amongst the most detrimental nonlinearities are stimulated Brillouin scattering (SBS), stimulated Raman scattering (SRS), and nonlinear refractive index $\mathrm{n}_{2}$-related wave-mixing phenomena (e.g., four-wave mixing, FWM, self-phase modulation, SPM). In order to mitigate these parasitic effects, the global optical fiber and laser community has developed micro-structured, large mode area (LMA) fibers whereby the fiber geometry is engineered to spread the optical power out over a larger effective area (i.e., core size). In addition to increasing the resultant complexity and cost of these fibers, such LMA designs introduce new parasitic phenomena, such as transverse mode instability (TMI), which presently serves as the dominant limitation in power scaling [2].

The approach adopted in this work is to attack these nonlinearities through the enabling materials from which they originate (i.e., the glass fiber core). Indeed, the Brillouin gain coefficient (BGC), the Raman gain coefficient (RGC), the thermo-optic coefficient (TOC) and the nonlinear refractive index $\left(\mathrm{n}_{2}\right)$ are all intrinsic material properties that respectively drive SBS, SRS, TMI and wave-mixing phenomena [3-6]. Consequently, this work focuses on developing and investigating core glass compositions for which the magnitude of these aforementioned properties can be reduced, enabling further power scaling.

As an illustrative example of the success of this approach, reductions of $\sim 20 \mathrm{~dB}$ and $\sim 3 \mathrm{~dB}$ in BGC and RGC, respectively, for sapphire-derived and YAG-derived all-glass aluminosilicate fibers were demonstrated relative to conventional silica fibers $[7,8]$. However, if each nonlinearity (e.g., SRS, SBS) can be individually reduced with the help of a given glass composition, this work investigates novel glasses that can diminish all nonlinearities simultaneously in a simple and scalable conventional circular core-cladding optical fiber geometry [9].

Fibers comprising an oxyfluoride core in the strontium fluoride $\left(\mathrm{SrF}_{2}\right)$ - alumina $\left(\mathrm{Al}_{2} \mathrm{O}_{3}\right)$ - silica $\left(\mathrm{SiO}_{2}\right)$ glass family, with a pure silica cladding, are fabricated and their properties discussed. Materially, $\mathrm{SrF}_{2}$ and $\mathrm{Al}_{2} \mathrm{O}_{3}$, when 
added to silica $\left(\mathrm{SiO}_{2}\right)$, participate in the formation of intrinsically low Brillouin and Raman scattering glasses. Further, the incorporation of fluorine, through $\mathrm{SrF}_{2}$, lessens the linear and nonlinear refractive indices $\left(\mathrm{n}, \mathrm{n}_{2}\right)$, as well as TOC, the later enabling consequent increase of TMI threshold. As for the realization of homogeneous glass cores, $\mathrm{Al}_{2} \mathrm{O}_{3}$ is incorporated into the silica matrix as it precludes phase separation that binary alkaline earth silicate systems typically exhibit in the high-silica region of the phase diagram. Additionally, active fibers were developed with the introduction of the ytterbium $(\mathrm{Yb})$ rare earth ion through the addition of minor amounts of $\mathrm{YbF}_{3}$ or $\mathrm{Yb}_{2} \mathrm{O}_{3}$, and their spectroscopic properties were studied.

The fibers were fabricated using the molten core method, as it is an efficient way to develop a large variety of fiber core glass compositions that could not be otherwise achieved using conventional chemical vapor deposition (CVD) techniques [10].

\section{FIBER FABRICATION AND RESULTS}

A precursor material (e.g., a mixture of $\mathrm{SrF}_{2}, \mathrm{Al}_{2} \mathrm{O}_{3}$ for a passive fiber) is introduced into a pure silica glass capillary preform ( 30 and $3 \mathrm{~mm}$ outer and inner diameters). The latter is then placed inside a draw tower furnace and heated to $2000{ }^{\circ} \mathrm{C}$. At this temperature, the silica cladding tube softens and the core precursor material melts. Silica from the surrounding cladding reacts with the molten core precursor materials and is subsequently incorporated into the core. The preform is thus directly drawn into a fiber with a target cladding diameter of $125 \mu \mathrm{m}$ (Fig. 1a). The fiber is coated during the draw with a conventional acrylate coating. During cooling as the fiber draws $\left(\sim 2000{ }^{\circ} \mathrm{C} / \mathrm{s}\right)$, the molten core is kinetically trapped into its glassy state, yielding to a graded-index silicate core $(\sim 85$ mole percent of $\mathrm{SiO}_{2}$ at the core center, the rest being the precursor materials) inside a silica cladding optical fiber, as exemplified in Fig. 1b. An important feature of these glasses is the reactivity of $\mathrm{SrF}_{2}$ with its molten silicate environment, resulting in its partial oxidation and consequent fluorine loss through the formation of volatile $\mathrm{SiF}_{4}$ species.
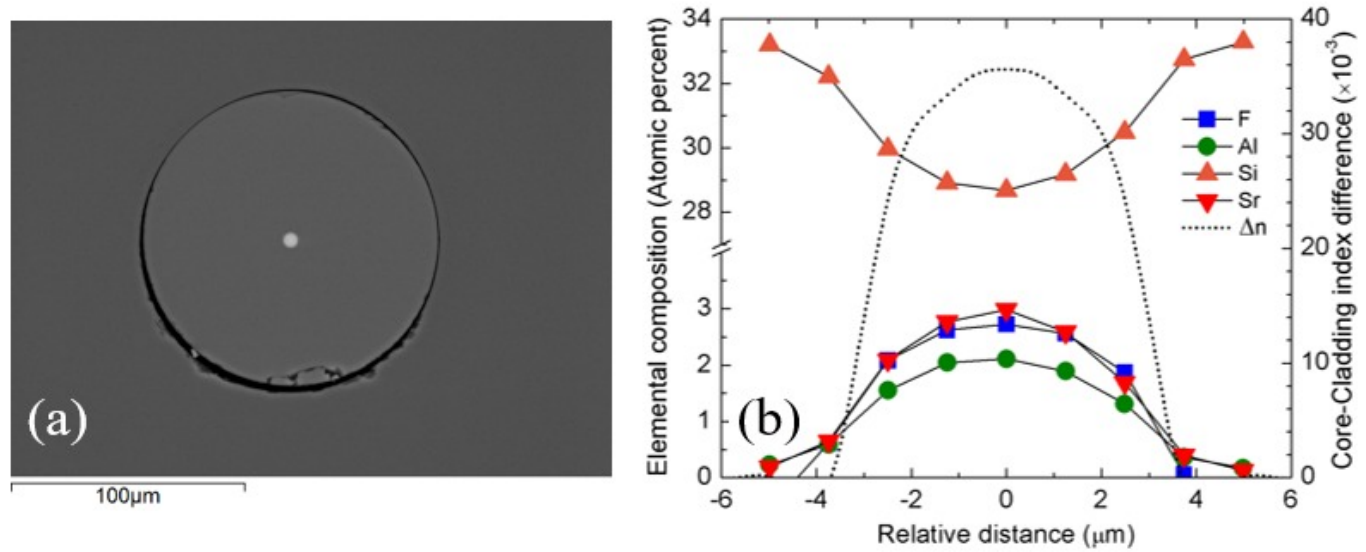

Fig. 1. a) Scanning electron microscope (SEM) micrograph of a strontium oxyfluoride core and silica cladding fiber [9]. b) Elemental composition (in atomic percent, At. \%) and core-cladding refractive index difference $(\Delta \mathrm{n})$ profiles as a function of the core radial distance $(\mu \mathrm{m})$. Not shown for reason of clarity is the concentration of oxygen, $\% \mathrm{O}$. However, $\% \mathrm{O}$ (in At. \%) $=100-[\% \mathrm{Si}+\% \mathrm{Al}+$ $\% \mathrm{Sr}+\% \mathrm{~F}]$.

Typical values of BGC, RGC, TOC and $\mathrm{n}_{2}$ for a series of strontium oxyfluoride-core fibers are reported in Table 1, and compared to conventional silica fibers. Relative to the latter, reductions of 6-8 dB, 1-2 dB, and 2-3 dB for BGC, $\mathrm{RGC}$, and TOC are measured, respectively, while $\mathrm{n}_{2}$ values are found to be within the range of silica fibers [11].

The low BGC values in these glass materials originate from the simultaneous contribution of multiple material properties that factor into the expression of BGC in optical fibers. Both $\mathrm{SrO}$ (from oxidation of some of the $\mathrm{SrF}_{2}$ ) and $\mathrm{Al}_{2} \mathrm{O}_{3}$ reduce the magnitude of the $\mathrm{p}_{12}$ transverse photoelastic constant of the multicomponent silicate glass core, but also increase the glass density $(\rho)$ and Brillouin scattering bandwidth $\left(\Delta v_{B}\right)$. As a result, the fabricated fibers exhibit considerably reduced BGC values. 
Table 1. Properties of fabricated oxyfluoride core fibers versus conventional silica fibers.

\begin{tabular}{ccc}
\hline Key properties & $\begin{array}{c}\text { Oxyfluoride } \\
\text { fibers }\end{array}$ & $\begin{array}{c}\text { Conventional } \mathrm{SiO}_{2} \\
\text { fibers }\end{array}$ \\
\hline $\mathrm{BGC}\left(\times 10^{-11} \mathrm{~m} / \mathrm{W}\right)$ & $0.3-0.6$ & 2.4 \\
$\mathrm{RGC}($ a. u. $)$ & $\sim 0.75$ & 1 \\
$\mathrm{TOC}\left(\times 10^{-6} / \mathrm{K}\right)$ & $4.9-6.3$ & 10.4 \\
$\mathrm{n}_{2}\left(\times 10^{-20} \mathrm{~m}^{2} / \mathrm{W}\right)$ & $\sim 3$ & $\sim 3$ \\
\hline
\end{tabular}

RGC is proportional to the peak intensity that dominates the Raman scattering bandwidth of the glass core materials. Although being multicomponent glasses, the fiber cores remain principally constituted of silica. Therefore, the dominating scattering peak is attributed to the Si-O-Si stretching modes situated around $440 \mathrm{~cm}^{-1}$ (Fig. 2a). Two principal contributions that lead to the reduction of RGC in the fabricated fibers are identified; a) a lower silica content in the glass cores that reduces the Si-O-Si peak strength, illustrated by the black arrow in Fig. 2a, and b) the minimized overlap among the different glass dopants' peaks present in the silicate network. The decrease of TOC is due to the increase in fluorine concentration ([F]), as illustrated in Fig. 2b. In these oxyfluoride glasses, the lower polarizability of the fluorine ions relative to the oxygen ions, coupled with the higher coefficient of thermal expansion (CTE) of the $\mathrm{SrF}_{2}$ glass compound, yields to intrinsically low TOC glass materials [12]. It is worth mentioning that at $[\mathrm{F}]=0$, the fiber exhibit lower TOC value than $\mathrm{SiO}_{2}$, driven by the large CTE of SrO. For completeness, $\mathrm{Al}_{2} \mathrm{O}_{3}$ exhibits a slightly higher TOC than $\mathrm{SiO}_{2}$ and therefore is not expected to participate in the reduction of TOC (glass core).
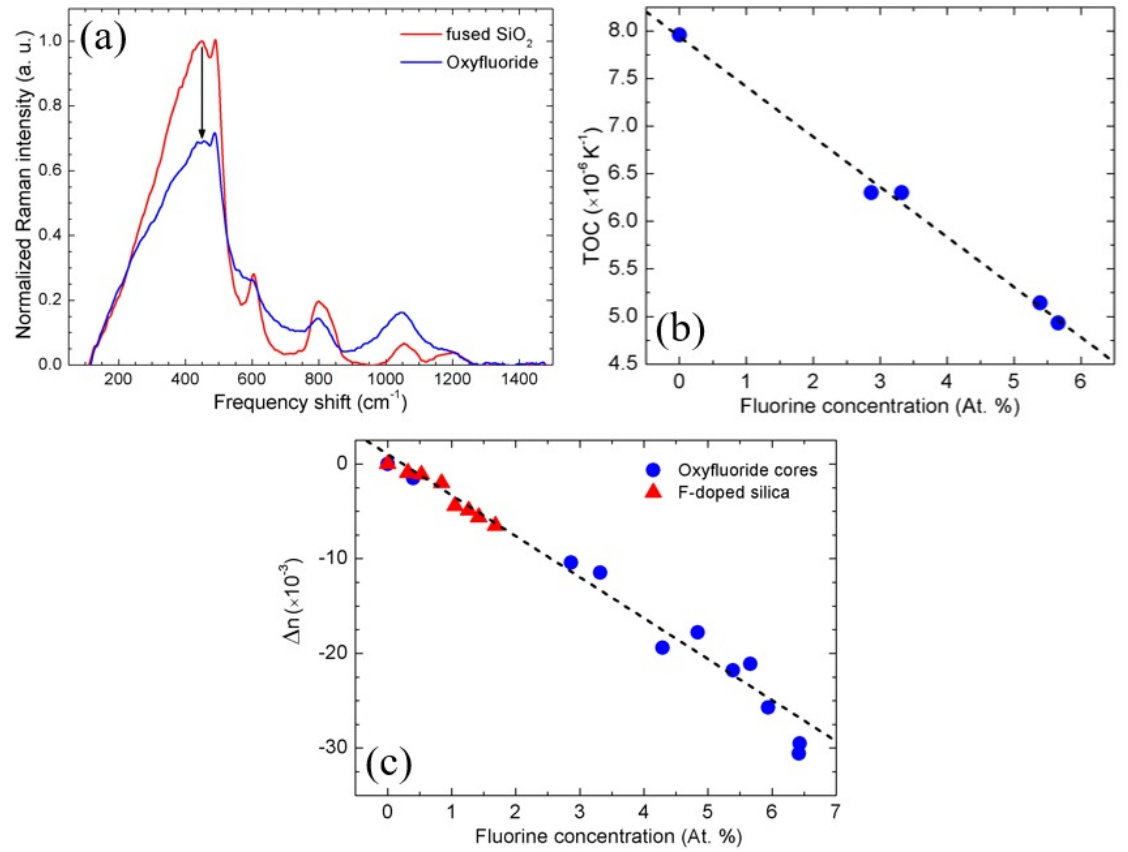

Fig. 2. a) Normalized and corrected Raman spectra of an oxyfluoride core (in red) and $\mathrm{SiO}_{2}$ (taken as a reference, in blue). b) Thermooptic coefficient (TOC) of oxyfluoride glass fibers as a function of fluorine concentration (in atomic percent, At. \%). c) Contribution of fluorine to the reduction of refractive index, relative to a non-containing fluorine glass analog. F-doped silica glass are taken from [13] as comparison.

The nonlinear refractive index, $\mathrm{n}_{2}$, of these oxyfluoride fibers is found to be of the same magnitude as pure $\mathrm{SiO}_{2}$ [9]. It is worth noting that glass compounds such as $\mathrm{SrO}$ and $\mathrm{Al}_{2} \mathrm{O}_{3}$ typically increase $\mathrm{n}_{2}$ values when added to silica. However, since fluorine and fluorides tend to mitigate this increase of $n_{2}$ [14], the developed oxyfluoride cores 
experience a nearly null change in $\mathrm{n}_{2}$ relative to $\mathrm{SiO}_{2}$. The same arguments can be used to discuss the influence of the aforementioned dopants on the linear refractive index (n), which factors into materials properties that contribute to optical nonlinearities. As can be observed in Fig. 2c, fluorine lowers the refractive index of the fiber core, in comparison of its non-containing fluorine glass analog. For completeness, using the molten core technique, fibers with $\sim 6$ At. $\%$ of fluorine were developed, which is 3 times higher than was can be achieved using conventional CVD techniques.

Table 2. Typical spectroscopic properties for three oxyfluoride core and silica cladding glass optical fibers.

\begin{tabular}{ccccc}
\hline Fibers & {$[\mathrm{F}]^{\mathrm{a}}$} & $\tau(\mu \mathrm{s})^{\mathrm{b}}$ & $\begin{array}{c}\lambda_{\mathrm{av}} \\
(\mathrm{nm})^{\mathrm{c}}\end{array}$ & $\sigma_{\mathrm{av}}\left(\times 10^{-20} \mathrm{~cm}^{2}\right)$ \\
\hline $\begin{array}{c}\text { Commercial } \\
\text { Yb:aluminosilicate }\end{array}$ & 0 & 743 & 1006.6 & 1.06 \\
1 & 0 & 1060 & 1004.9 & 1.09 \\
2 & 2.35 & 1240 & 1001.6 & 0.90 \\
3 & 4.42 & 1270 & 999.2 & 0.85 \\
\hline
\end{tabular}

a: in atomic percent (At. \%).

${ }^{\mathrm{b}}:$ measured for a decrease of 1 in the $\log$ scale.

c: defined as $\lambda$ (average) $=\int \lambda \times \mathrm{I}(\lambda) \times \mathrm{d} \lambda / \int \mathrm{I}(\lambda) \times \mathrm{d} \lambda$.

d: defined as $\sigma($ average $)=\int \sigma \times \mathrm{I}(\sigma) \times \mathrm{d} \sigma / \int \mathrm{I}(\sigma) \times \mathrm{d} \sigma$.

Ytterbium spectroscopic properties for three fabricated active fibers with different fluorine concentrations, along with a typical commercially available Yb-doped aluminosilicate (Yb:AS) fiber are provided in Table 2. Their normalized emission cross sections are displayed in Fig. 3. The increase of the radiative lifetime $(\tau)$, and the decrease of both averaged emission wavelength $\left(\lambda_{\mathrm{av}}\right)$ and cross section $\left(\sigma_{\mathrm{av}}\right)$ as a function of fluorine concentration are characteristic of fluoride glasses [15]. This is particularly interesting for fiber laser designers as these compositions offer great property tunability, including "fluoride-like" properties, while preserving the mechanical strength and robustness of silica fibers. Materially, these strong changes of the spectroscopic properties as a function of fluorine concentration suggests the fluorine ion to be surrounding the $\mathrm{Yb}$ ion.

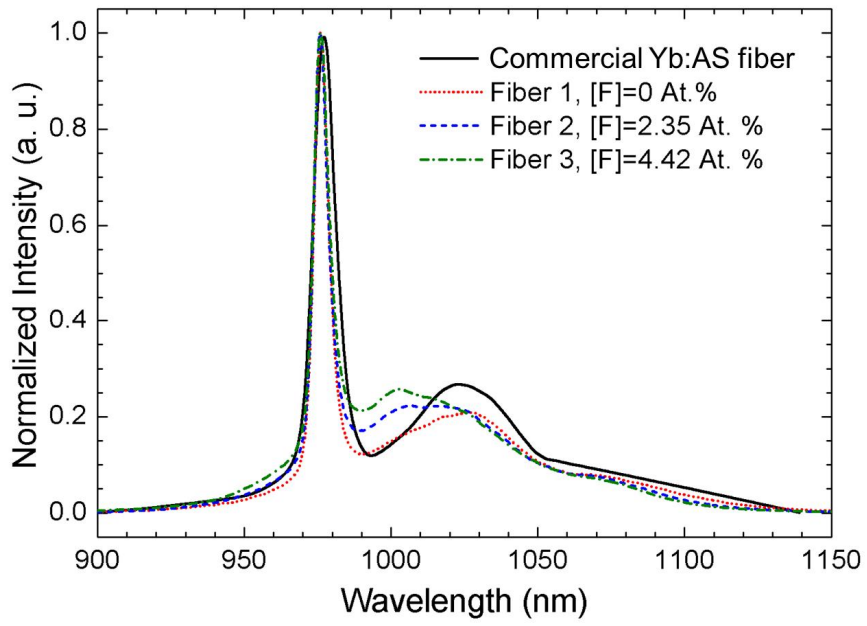

Fig. 3. Normalized emission spectra of fibers in Table 2.

From a laser performance standpoint, the $\tau \times \sigma_{\mathrm{av}}$ figure of merit of was found $\sim 35-45 \%$ higher than typical (commercial) aluminosilicate fibers. This, coupled with the lower quantum defect (QD) available from these multicomponent fluorosilicate fibers $(<1.5 \%)$ relative to conventional silica and silicate fibers $(\sim 5 \%)$ [16], is expected to considerably 
improve laser performance. It is worth mentioning that the low TOC values $(\sim 3 \mathrm{~dB})$, along with the low $\mathrm{QD}$, are also expected to further prevent parasitic thermal effects (e.g., thermal lensing).

\section{CONCLUSIONS}

The unified materials approach advocated herein is found to be an efficient route in mitigating optical nonlinearities in glass optical fibers and paves the way to further power scaling. The glass oxyfluoride core and silica cladding glass optical fibers fabricated using the molten core method exhibit reduced BGC, RGC, TOC relative to conventional silica fibers, while preserving low linear and nonlinear refractive indices. Moreover, spectroscopic properties of active (i.e., $\mathrm{Yb}$-doped) fibers suggest high efficiency and enhanced lasing performances using these core compositions, adequate for high energy laser applications. Future work focuses on the reduction of attenuation losses (currently in the $\mathrm{dB} / \mathrm{m}$ range) through the use of higher purity precursor materials.

\section{REFERENCES}

[1] Zervas, M. N., and Codemard, C. A., "High Power Fiber Lasers: A Review," IEEE J. Sel. Top. Quantum Electron. 20, 1-23 (2014).

[2] Dong, L., "Stimulated thermal Rayleigh scattering in optical fibers," Opt. Express 21, 915-918 (2013).

[3] Ballato, J., Cavillon, M., and Dragic, P. D., "A Unified Materials Approach to Mitigating Optical Nonlinearities in Optical Fiber. I. Thermodynamics of Optical Scattering," Int. J. Appl. Glas. Sci. (in press, 2018).

[4] Dragic, P. D., Cavillon, M., Ballato, A., and Ballato, J., "A Unified Materials Approach to Mitigating Optical Nonlinearities in Optical Fiber. II. A. Material Additivity Models and Basic Glass Properties," Int. J. Appl. Glas. Sci. (in press, 2018).

[5] Dragic, P. D., Cavillon, M., Ballato, A., and Ballato, J., "A Unified Materials Approach to Mitigating Optical Nonlinearities in Optical Fiber. II. B. The Optical Fiber, Material Additivity and the Nonlinear Coefficients," Int. J. Appl. Glas. Sci. (in press, 2018).

[6] Cavillon, M., Kucera, C., Hawkins, T., Dawson, J., Dragic, P. D., and Ballato, J. "A Unified Materials Approach to Mitigating Optical Nonlinearities in Optical Fiber. III. Canonical Systems and Results," Int. J. Appl. Glas. Sci. (in press, 2018).

[7] Dragic, P. D., Hawkins, T., Foy, P., Morris, S., and Ballato, J., "Sapphire-derived all-glass optical fibres," Nat. Photonics 6, 629-635 (2012).

[8] Dragic, P. D., and Ballato, J., "Characterization of the Raman gain spectra in Yb:YAG-derived optical fibers," Electron. Lett. 49, 895-897 (2013).

[9] Cavillon, M., Kucera, C., Hawkins, T., Runge, A. F. J., Peacock, A. C., Dragic, P. D., and Ballato, J., "Oxyfluoride core silica-based optical fiber with intrinsically low nonlinearities for high energy laser applications," J. Light. Technol. (in press, 2018).

[10]Dragic, P. D., and Ballato, J., "Rethinking Optical Fiber: New Demands, Old Glasses," J. Am. Ceram. Soc. 96, 2675-2692 (2013).

[11]Kato, T., Suetsugu, Y., and Nishimura, M., "Estimation of nonlinear refractive index in various silica-based glasses for optical fibers," Opt. Lett. 20, 2279 (1995).

[12] Prod'homme, L., "A new approach to the thermal change in the refractive index of glasses," Phys. Chem. Glas. 1, $119-122(1960)$.

[13] Koike A., and Sugimoto, N., "Temperature dependences of optical path length in fluorine-doped silica glass and bismuthate glass," 6116, 1-8 (2006).

[14] Weber, M., Milam, D., and Smith, W., "Nonlinear refractive index of glasses and crystals," Opt. Eng. 17, 463-469 (1978).

[15] Weber, M., Lynch, J., Blackburn, D., and Cronin, D., "Dependence of the stimulated emission cross section of $\mathrm{Yb}^{3+}$ on host glass composition," Quantum Electron. IEEE J. 19, 1600-1608 (1983).

[16] Yu, N., Cavillon, M., Kucera, C., Hawkins, T., Ballato, J., and Dragic, P. D., "Low Quantum Defect Fiber Lasers via $\mathrm{Yb}$-Doped Multicomponent Fluorosilicate Optical Fiber," Conference on Lasers and Electro-Optics (CLEO), OSA Technical Digest (in press, 2018). 